\title{
Selection of lactic acid bacteria producing antimicrobial strain such the genus lactococcus isolated from algerian raw goat's milk
}

\author{
Benhamouche N., ${ }^{1 *}$, Talhi M. ${ }^{2}$, Kihal M. ${ }^{1}$ \\ ${ }^{1}$ Laboratory of Applied Microbiology Department of Biology, Faculty of Sciences, Oran University -Es-Senia \\ ${ }^{2}$ Molecular Biology Laboratory, Department of Applied Molecular Genetics, Faculty of Science, University USTO-MB
}

\section{Email address:}

norabenhamouche@yahoo.fr (B. N.)

\section{To cite this article:}

Benhamouche N. , Talhi M. , Kiha M. . Selection of Lactic Acid Bacteria Producing Antimicrobial Strain such the Genus Lactococcus Isolated from Algerian Raw Goat'S Milk. International Journal of Nutrition and Food Sciences. Vol. 1, No. 1, 2012, pp. 23-32. doi: $10.11648 /$ j.jijnfs.20120101.13

\begin{abstract}
Lactic acid bacteria are known for their ability to produce inhibitory substances against unwanted germs involved in food poisoning such as Listeria innocua. Species of lactic acid bacteria can inhibit harmful germs subject of this work. techniques confrontation on solid medium and the effect of the substance on the growth of Listeria innocua were performed. The experience was conducted bacterial antagonism in the solid medium by the method of double layer and method of wells, the whole experience was packaged in a buffered medium in order to eliminate the effect of acidity . 7 strains that isolates gave an antagonistic action against Listeria innocua. The strain of Lactococcus lactis (8b), isolated from raw goat's milk showed an anti-listeria in vitro and was selected because of its ability to inhibit as Staphylococcus aureus. With a diameter of $15 \mathrm{~mm}$, the action of proteolytic enzymes, trypsin, chymotrypsin is shown that the substance was protein in nature, the kinetics of growth in milk medium showed that the number of St. aureus after $24 \mathrm{~h}$ of incubation was reduced to 7.68 log cfu in mixed culture with strain $8 \mathrm{~b}$ which was comparable to the control of $9.14 \mathrm{log} \mathrm{cfu}$, which signifies that the survival rate was 3.4\%, the phenotypic (biochemical and physiological) and molecular-based ADNr16Ss showed that the strain is Enterococcus faecium, duran, hirae. The sequencing results showed that Leuconostoc mesenteroides and Enterococcus faecium have summers dominant anti-Listeria species in milk samples from Algerian goat. Isolates had the potential of multiple bacteriocin production and do not have some important virulence. Importance and impact of the study: The Enterococci in milk in this region of western Algeria could be partly responsible for the safety of cheese and could be useful for the production of anti-Listeria cultures protection. The purpose of the study: Our study's main objective is the selection ,and study phenotypic and molecular lactic acid bacteria from Lactococcus genus possessing biotech traits such as the production of new inhibitory substance and the study of the interaction of Listeria innocua screw opinion and St. aureus that both methods were used, a traditional approach based on morphological and biochemical studies of different cultural characteristics and a method of direct molecular amplification of bacterial DNA using PCR / RAPD colony on having targeted DNA fragment 16S lactic acid bacteria using the two primers universal: 20F (5'AGAGTTTGATCATGGCTCAG-3 '). 1500R (5'-GGTTACCTTGTTACGACTT-3 ') and two specific primers OPA-3 (5'-AGTCAGCCAC - 3') and OPH 3 (5'-AGACGTCCAC-3 ') (Bioprobe, France).
\end{abstract}

Keywords: Bacteriocin, Listeria, St. Aureus, Goat's Milk; Adnr16s, PCR / RAPD

\section{Introduction}

Lactic acid bacteria possess remarkable technological advantages for bio food preservation (Ananou.S, 2010, Kouakou Privat, Philippe Thonart2011), thanks to the production of a wide variety of antimicrobial substances (compounds antagonists) which prevent the growth of pa- thogens transmitted by food (Listeria, Staphylococcus, ( Labioui., 2005, Gravesen A et al 2011). The main factor responsible for the inhibition in fermented foods is the production of organic acids and the concomitant decrease in $\mathrm{pH}$. Moreover, lactic acid bacteria can secrete inhibitors in the middle specific and which are the most interesting technological standpoint due to their nature protein (Thonart and Dortu, 2009). inhibitors are known bacteriocin. Lactic 
acid bacteria produce a variety of peptides or proteins having activity antibacterial. called bacteriocins These molecules could be better used in dairy various transformations, notably to ensure the hygienic safety of certain cheeses. (Bayoub et al., 2006 Dortu, 2008). bacteriocins represent a large class of antagonistic substances which vary considerably in terms of their molecular weight, their biochemical properties, their spectrum and their mode of action (Dortu 2008). All bacteriocins produced by lactic acid bacteria described so far have activity against Gram-positive bacteria. however, the modes of action of bacteriocins on the membrane are varied (Dortu, 2008). Currently a new generation of vaccine is being developed using strains of lactic acid bacteria ( Touvart 2009, M.Zadeh 2009).

\section{Materials and Methods}

\subsection{Bacterial Strains and Culture Conditions}

Lactococcus lactis subsp lactis was isolated from raw goat milk from Algeria. Stored at $-20^{\circ} \mathrm{C}$ in a medium containing $10 \%$ milk $(0.05 \%$ enriched yeast extract $)$ and $30 \%$ glycerol deposited at a temperature of $\left(-20^{\circ} \mathrm{C}\right)$. As the need arises, the cultures are thawed quickly in milk and subcultured twice before use (Samelis et al, 1998). St. aureus, Listeria, Bacillus and E. coli were stored at $-20^{\circ}$ passaged several times in the liquid medium at $37^{\circ} \mathrm{C}$ soybean strain was subcultured inhibitory several times in MRS medium and M17 liquid at $30^{\circ} \mathrm{C}$.

Listeria innocua strain (S71 and LMBA St.aureus CIP20256) of ENITA Bordeaux, St. aureus (ATCC.602 laboratory Tlemcen). the research activity inter bacterial antagonist interaction was performed by the direct method: according to Fleming et al., (1975), is to cultivate both strains in the same medium double layer and the indirect method described by Barefoot and al. (1983), that use the supernatant of the culture medium of a bacterium assumed to be inhibitory. The action of the supernatant obtained after centrifugation of the culture medium is tested against a bacterium called sensitive indicator. St. aureus strains, Bacillus Listeria and E. coli were used in both cases come indicator strains. Acidity factor was removed by the use of a buffered medium (0.2 M phosphate buffer $\mathrm{pH} 7)$. To detect the production of $\mathrm{H} 2 \mathrm{O} 2$, is carried out in the presence of crop in an amount of catalase $1 \mathrm{mg} / \mathrm{ml}$ medium. The enzyme and the indicator strain were mixed in soft agar supercooled. After incubation reading the results is compared to the control containing no catalase.

\subsection{Search Lysogenic Phages}

With a Pasteur pipette was cut a piece of agar in the zone of inhibition.

This fragment was suspended in $1 \mathrm{ml}$ of sterile medium containing $50 \mathrm{mu} .1$ of chloroform. After stirring, allowed to settle 5 minutes, then was taken $300 \mu$ l of medium that is was added to $7 \mathrm{ml}$ of soft agar containing the indicator strain. The mixture was poured aseptically into a petri dish and incu- bated for $48 \mathrm{~h}$ at $28^{\circ} \mathrm{C}$. The presence of lysis plaque indicates the presence of phage.

\subsection{Search Inhibitions Diffusion in Solid}

Filtrate was concentrated to $100 \mu$ l of acetone are placed in wells made previously in M17 medium buffered solid. Deposits are made as follows: Well 1: control (untreated float) Well 2: + trypsin filtrate, Well 3 + filtrate chymotrypsin, Wells $4+$ filtrate lysozyme, Well 5: filtrate heated to $100^{\circ} \mathrm{C}$ for $30 \mathrm{~min}$., Wells 6 filtrate $\mathrm{pH} 7$, Well 7: concentrated filtrate with chloroform.

After a one hour incubation at room temperature, allowing the diffusion potential antimicrobial substances. Petrie boxes are covered with $7 \mathrm{ml}$ of soft agar containing the indicator strain. : Following a second incubation of 24 hours.

\subsection{Growth Kinetic and Acidification}

The evaluation of the acidity produced by a pure strains was performed by titration and $\mathrm{pH}$ - metritis. Each strain was inoculated into $10 \mathrm{ml}$ sterile skim milk. Following incubation until coagulation of milk at $28^{\circ} \mathrm{C}$ for $18 \mathrm{~h}$ or more (there was clotting time), everything is transferred into sterile $200 \mathrm{ml}$ skimmed milk and homogenized. the mixture was distributed into sterile tubes due $10 \mathrm{ml} /$ tube and the kinetics of growth and acidification were performed simultaneously have the following time intervals: $0 \mathrm{~h}, 3 \mathrm{~h}, 6 \mathrm{~h}, 9 \mathrm{~h}, 14 \mathrm{~h}, 16 \mathrm{~h}$, $24 \mathrm{~h}, 48 \mathrm{~h}, 72 \mathrm{~h}, 96 \mathrm{~h}$. For this study we used a strain of lactic acid bacteria strain as inhibitory considered effective strain and a pathogenic strain as Staphylococcus aureus strain test laboratory of Tlemcen ATCC.602 that the reason inoculate 103 - $105 \mathrm{cfu} / \mathrm{ml}$ and according Hamama al., 2003), we calculated the evolution of a count from $0 \mathrm{~h}$ after $18 \mathrm{~h}$ preculture of each strain and strain inhibitory test and mixed culture.

\subsection{Random Amplified Polymorphic DNA (RAPD)}

The random amplified polymorphic deoxyribonucleic acid (Random Amplified Polymorphic DNA) is performed directly on colony strains étudiées.Cette amplification of DNAs is performed in a thermal cycler (PTC-200, MJ Research, USA) with the program starting with a initial denaturation step at $94^{\circ} \mathrm{C}$ durant 5 min, followed by 45 cycles of amplification of the DNAs with three stages that are $30 \mathrm{~s}$ at $94^{\circ} \mathrm{C}, 1 \mathrm{~min} 30 \mathrm{~s}$ at $36^{\circ} \mathrm{C}$ and $1 \mathrm{~min} 30 \mathrm{~s}$ at $72^{\circ} \mathrm{C}$. Immediately after the last cycle of amplification, the reaction mixture was kept at $72^{\circ} \mathrm{C}$ for $10 \mathrm{~min}$ to complete the amplification possible. DNA fragments amplified by PCR were then separated by electrophoresis voltage 5 V.cm-1 agarose gel $(1.5 \%$ [w / v] added BET [ethidium bromide] to reason of $0.1 \mathrm{mg}$ per $300 \mathrm{~mL}$ of agarose gel) and then immersed in TBE buffer ( $89 \mathrm{mM}$ Tris, $89 \mathrm{mM}$ boric acid, $2 \mathrm{mM}$ EDTA). Amplification without DNA (negative control) is carried out in each series to control possible contamination of foreign DNAs in the matrix. Reading and photography electrophoretic profiles are performed under ultra violet (UV) transilluminator with (Vilber Lourmat, Allemange) at $254 \mathrm{~nm}$. The 
size of the DNA fragments was estimated by comparison with standard DNA marker $\lambda$ EcoRI / HindIII Digest (Q-biogenic) (Appendix 2). Polymorphic electrophoretic profiles are exploited by comparing the DNA bands on agarose gel. Twenty microliters of the PCR product are sent to Genome Express (France) for sequencing.

The sequences are realized in the genome called Express began, and it is as follows: partial DNA sequences of the PCR products (the first 600 bases) were determined using a Taq Dye Deoxy terminator sequence kit cycle ( Perkin-Elmer, Foster City, CA, USA) and the protocol recommended by the supplier. Products of the sequencing reaction were analyzed with a model 373A automated DNA sequencer (Applied Biosystems, Foster City, CA, USA). Databases (GenBank) looked for sequences similar to the $16 \mathrm{~S}$ rDNA sequence obtained.

\section{Results and Discussion}

Among the 48 strains confrontations between 768 and according to the percentage (\%) inhibition was able to get 460 cases or $60 \%$ inhibition. These results were obtained using a medium is not buffered. In contrast, there was a small number, 130 cases of inhibition, in the middle $16 \%$ buffered. Of the 48 strains, 36 strains possess an inhibitory power and 12 strains showed no inhibition in buffered medium (see Table 1).

According to the percentage of inhibition is buffered medium 20 strains were selected (16 and 4 inhibitory indicator strains (see Table 2).

Table 2. Percentage (\%) inhibition of various confrontations OF inhibitory and indicator

\begin{tabular}{|c|c|c|c|c|c|c|}
\hline \multirow{2}{*}{$\begin{array}{l}\text { CODE } \\
\text { Inhibitric }\end{array}$} & \multicolumn{2}{|c|}{$\begin{array}{l}(\%) \text { d'inhibition } \\
\text { Série } 1\end{array}$} & \multicolumn{2}{|c|}{$\begin{array}{l}\text { (\%) d'inhibition } \\
\text { Série } 2\end{array}$} & \multicolumn{2}{|c|}{$\begin{array}{l}(\%) \text { d'inhibition } \\
\text { Série } 3\end{array}$} \\
\hline & eDs53 & $42 \%$ & $18 \mathrm{~b}$ & $18 \%$ & $2 b$ & $18 \%$ \\
\hline \multicolumn{2}{|c|}{ Inhibitrice $12 \mathrm{~b}$} & $42 \%$ & T16 & $48 \%$ & $20 \mathrm{~b}$ & $42 \%$ \\
\hline \multicolumn{2}{|c|}{ Inhibitrice 11a } & $42 \%$ & $1 b$ & $72 \%$ & Ds 15 & $42 \%$ \\
\hline \multicolumn{2}{|c|}{ Inhibitrice Ds 16} & $36 \%$ & & & $8 \mathrm{~b}$ & $24 \%$ \\
\hline \multicolumn{2}{|c|}{ Inhibitrice $7 b$} & $30 \%$ & & & $30 \mathrm{a}$ & $72 \%$ \\
\hline \multicolumn{2}{|c|}{ inhibitrice $4 a$} & $42 \%$ & & & $17 \mathrm{a}$ & $34 \%$ \\
\hline \multicolumn{3}{|c|}{ Inhibitrice } & & & Ds13 & $24 \%$ \\
\hline \multicolumn{2}{|c|}{ indicatrice $24 a$} & $42 \%$ & $\mathrm{~T} 20$ & $54 \%$ & & \\
\hline \multicolumn{2}{|c|}{ Indicatrice } & & Ds44 & $42 \%$ & & \\
\hline \multicolumn{3}{|c|}{ indicatrice } & Ds14 & $24 \%$ & & \\
\hline
\end{tabular}

Table 1. percentage inhibition (INH) inter bacterial environment Buffered (MT) and unbuffered (MNT) for 16 strains by the method directly.

\begin{tabular}{|c|c|c|c|c|c|}
\hline & $\begin{array}{l}\text { Code des } \\
\text { souches }\end{array}$ & $\begin{array}{l}\text { INH/16 } \\
\text { MNT }\end{array}$ & $\begin{array}{l}\text { INH/16 } \\
\text { MT }\end{array}$ & $\begin{array}{l}\% \\
\text { MNT }\end{array}$ & $\begin{array}{l}\% \\
\text { MT }\end{array}$ \\
\hline \multirow{23}{*}{ Série 1} & $7 b$ & 16 & 5 & 100 & 30 \\
\hline & $11 \mathrm{a}$ & 16 & 7 & 100 & 42 \\
\hline & $7 a$ & 16 & 1 & 100 & 6 \\
\hline & Ds53 & 16 & 7 & 100 & 42 \\
\hline & Ds 16 & 14 & 6 & 84 & 36 \\
\hline & $12 b$ & 15 & 7 & 90 & 42 \\
\hline & $4 a$ & 15 & 7 & 90 & 42 \\
\hline & $3 a$ & 15 & 1 & 90 & 6 \\
\hline & $21 \mathrm{a}$ & 16 & 0 & 100 & 0 \\
\hline & $18 \mathrm{a}$ & 15 & 1 & 90 & 6 \\
\hline & Ds8 & 0 & 0 & 0 & 0 \\
\hline & $25 a$ & 15 & 1 & 90 & 6 \\
\hline & $24 \mathrm{a}$ & 16 & 0 & 100 & 0 \\
\hline & Ds44 & 16 & 0 & 100 & 0 \\
\hline & $35 \mathrm{~T}$ & 15 & 0 & 90 & 0 \\
\hline & $34 b$ & 15 & 2 & 90 & 12 \\
\hline & Ds14 & 1 & 0 & 6 & 0 \\
\hline & $22 a$ & 11 & 2 & 66 & 12 \\
\hline & T16 & 11 & 8 & 66 & 48 \\
\hline & $\mathrm{T} 20$ & 11 & 2 & 66 & 12 \\
\hline & $6 a$ & 10 & 1 & 60 & 6 \\
\hline & Ds36 & 0 & 0 & 0 & 0 \\
\hline & $11 \mathrm{~b}$ & 0 & 0 & 0 & 0 \\
\hline \multirow{16}{*}{ Série 2} & Ds37 & 11 & 1 & 66 & 6 \\
\hline & $18 \mathrm{~b}$ & 13 & 3 & 98 & 18 \\
\hline & $\mathrm{T} 35$ & 13 & 0 & 96 & 0 \\
\hline & Ds39 & 0 & 1 & 0 & 6 \\
\hline & $1 b$ & 11 & 12 & 66 & 72 \\
\hline & Ds 18 & 7 & 2 & 42 & 12 \\
\hline & Ds51 & 0 & 0 & 0 & 0 \\
\hline & $31 b$ & 4 & 2 & 24 & 12 \\
\hline & $25 b$ & 10 & 0 & 60 & 0 \\
\hline & Ds13 & 12 & 4 & 72 & 24 \\
\hline & Ds29 & 0 & 1 & 0 & 6 \\
\hline & $29 b$ & 11 & 2 & 66 & 12 \\
\hline & $27 b$ & 5 & 1 & 30 & 6 \\
\hline & $28 \mathrm{~b}$ & 13 & 2 & 78 & 12 \\
\hline & Ds52 & 15 & 1 & 90 & 6 \\
\hline & $17 \mathrm{a}$ & 0 & 4 & 0 & 34 \\
\hline \multirow[t]{9}{*}{ Série 3} & $16 \mathrm{a}$ & 15 & 0 & 90 & 0 \\
\hline & Ds 15 & 1 & 7 & 6 & 42 \\
\hline & $8 b$ & 13 & 4 & 78 & 24 \\
\hline & $19 a$ & 0 & 1 & 0 & 6 \\
\hline & $30 \mathrm{a}$ & 12 & 12 & 72 & 72 \\
\hline & $2 b$ & 9 & 3 & 54 & 18 \\
\hline & $\mathrm{T} 24$ & 12 & 1 & 72 & 6 \\
\hline & $20 \mathrm{~b}$ & 13 & 7 & 78 & 42 \\
\hline & Ds23 & 0 & 2 & 0 & 12 \\
\hline
\end{tabular}

The 20 strains that were selected were reidentified by physiological and biochemical testes (see Table3). 
Table 3. Physiological and biochemical character strains

\begin{tabular}{|c|c|c|c|c|c|c|c|c|c|c|c|c|c|c|c|c|c|c|c|c|}
\hline & 12 & 2 & 3 & 4 & 5 & 6 & 7 & 8 & 9 & 10 & 11 & 12 & 13 & 14 & 15 & 16 & 17 & 18 & 19 & 20 \\
\hline $\begin{array}{l}\text { Leuconostoc } \\
\text { mesenteroides } \\
\text { subsp.mesenteroides }\end{array}$ & $2 b+$ & & - & - & + & + & + & + & + & - & + & + & + & + & + & + & + & + & + & + \\
\hline $\begin{array}{l}\text { Lactococcus lactis sub } \\
\text { sp lactis biovar diacetylactis }\end{array}$ & $1 b+$ & & - & + & + & + & - & + & + & - & + & + & + & + & + & - & - & + & - & - \\
\hline $\begin{array}{l}\text { Lactococcus lactis sub } \\
\text { sp lactis biovar diacetylactis }\end{array}$ & $20 \mathrm{~b}+$ & & - & + & + & + & - & + & + & - & + & + & + & + & + & - & - & + & - & - \\
\hline $\begin{array}{l}\text { Lactococcus lactis sub sp } \\
\text { lactis biovar diacetylactis }\end{array}$ & $\begin{array}{l}\text { Ds } \\
15\end{array}$ & & - & + & + & + & - & + & + & - & + & + & + & + & + & - & - & + & - & - \\
\hline Lactococcus lactis sub sp lactis & $8 b+$ & + & - & - & - & + & - & + & + & - & + & + & + & + & + & - & - & + & - & - \\
\hline $\begin{array}{l}\text { Lactococcus lactis sub sp } \\
\text { lactis biovar diacetylactis }\end{array}$ & $30 \mathrm{a}+$ & & - & + & + & + & - & + & + & - & + & + & + & + & + & - & - & + & - & - \\
\hline Lactococcus lactis sub sp lactis & $4 a+$ & & - & - & - & + & - & + & + & - & + & + & + & + & + & - & - & + & - & - \\
\hline Lactococcus lactis sub sp lactis & $\begin{array}{l}\text { Ds } \\
13\end{array}$ & & - & - & - & + & - & + & + & - & + & + & + & + & + & - & - & + & - & - \\
\hline Streptococus thermophilus & $12 \mathrm{~b}+$ & & - & - & - & - & + & + & - & - & + & + & - & - & - & - & + & - & + & - \\
\hline Streptococus thermophilus & $11 \mathrm{a}+$ & & - & - & - & - & + & + & - & - & + & + & - & - & - & + & + & - & - & - \\
\hline Lactococcus lactis sub sp lactis & $\begin{array}{l}\text { Ds } \\
16\end{array}$ & & - & - & - & + & - & + & + & - & + & + & + & + & + & - & - & + & - & - \\
\hline Enterococcus sp & $7 b+$ & + & - & - & - & + & - & + & + & + & + & + & + & + & + & + & + & + & + & - \\
\hline Lactococcus lactis sub sp lactis & $\begin{array}{l}\mathrm{T} 1 \\
6\end{array}$ & + & - & - & - & + & - & + & + & - & + & + & + & + & + & - & - & + & - & - \\
\hline Enterococcus sp & $17 \mathrm{a}+$ & & - & - & - & + & - & + & + & + & + & + & + & + & + & + & + & + & + & - \\
\hline Streptococus thermophilus & $18 \mathrm{~b}+$ & & - & - & - & - & + & + & - & - & + & + & - & - & - & + & + & - & + & - \\
\hline Lactococcus lactis sub sp lactis & $\begin{array}{l}\text { Ds } \\
53\end{array}+$ & & - & - & - & + & - & + & + & - & + & + & + & + & + & - & - & + & - & - \\
\hline $\begin{array}{l}\text { Lactococcus lactis sub } \\
\text { sp lactis biovar diacetylactis }\end{array}$ & $24 \mathrm{a}+$ & & - & + & + & + & - & + & + & - & + & + & + & + & + & - & - & + & - & - \\
\hline $\begin{array}{l}\text { Leuconostoc } \\
\text { mesenteroides } \\
\text { subsp.mesenteroides }\end{array}$ & $\begin{array}{l}\mathrm{T} 2 \\
0\end{array}$ & & - & + & + & + & - & + & + & - & + & + & + & + & + & + & + & + & + & + \\
\hline Pediococcus acidilacticii & $\begin{array}{l}\text { Ds } \\
44\end{array}$ & & - & - & - & + & - & + & + & - & + & + & + & + & + & + & + & + & + & - \\
\hline Lactococcus lactis sub sp lactis & $\begin{array}{l}\text { Ds } \\
14\end{array}$ & & - & - & - & + & + & + & + & - & + & + & + & - & - & + & - & + & + & + \\
\hline
\end{tabular}

$1=$ code of strain, $2=$ Gram, $3=$ catalase, $4=$ citrate, $5=$ acetoine, $6=$ Arginine, $7=$ dextrane, $8=$ croissance àpH(9.2), $9=$ croissanceNacl(4\%), $10=$ croissanceNacl( $6,5 \%), 11=$ Glucose, $12=$ Lactose, $13=$ Galactose, $14=$ Maltose, $15=$ Manitol, 16=Raffinose, $17=$ Saccaros e, $18=$ Xylose, $19=$ Arabinose, $20=$ Production du gaz. 
Table 4. Interaction between bacteria Gram + and Gram-

\begin{tabular}{|c|c|c|c|c|c|c|c|c|}
\hline \multirow{3}{*}{ Nom espèce } & \multirow{3}{*}{ Code } & $\begin{array}{l}\text { St. aureus } \\
\text { ATCC.602(TLEMCEN) }\end{array}$ & $\begin{array}{l}\text { St.aureus } \\
\text { Cip20256(BORDEAUX) }\end{array}$ & $\begin{array}{r}\text { Listeria } \\
\text { inoccua }\end{array}$ & & E.coli & $\begin{array}{l}\text { Bacillus } \\
\text { cereus }\end{array}$ & 24a indicatrice \\
\hline & & \multicolumn{7}{|c|}{ Diamètre de la zone d'inhibition (mm) } \\
\hline & & MRS & MRS & MRS & M17 & MRS & MRS & MRS \\
\hline $\begin{array}{l}\text { Leuconostoc } \\
\text { mesenteroides } \\
\text { subsp.mesenteroides }\end{array}$ & $2 b$ & 05 & 0 & - & - & 0 & 0 & 08 \\
\hline $\begin{array}{l}\text { Lactococcus lactis } \\
\text { sub sp lactis } \\
\text { biovar diacetylactis }\end{array}$ & $1 \mathrm{~b}$ & 11 & 0 & 12 & 10 & 0 & 0 & 12 \\
\hline $\begin{array}{l}\text { Lactococcus lacti } \\
\text { s sub sp lactis } \\
\text { biovar diacetylactis }\end{array}$ & $20 \mathrm{~b}$ & 12 & 0 & 13 & 11 & 0 & 0 & 14 \\
\hline $\begin{array}{l}\text { Lactococcus lactis } \\
\text { sub sp lactis } \\
\text { biovar diacetylactis }\end{array}$ & Ds15 & 04 & 0 & - & - & 0 & 0 & 03 \\
\hline $\begin{array}{l}\text { Lactococcus lactis } \\
\text { sub sp lactis }\end{array}$ & $8 b$ & 15 & 0 & 14 & 11 & 0 & 0 & 13 \\
\hline $\begin{array}{l}\text { Lactococcus lactis } \\
\text { sub sp lactis } \\
\text { biovar diacetylactis }\end{array}$ & $30 \mathrm{a}$ & 08 & 0 & - & - & 0 & 0 & 11 \\
\hline $\begin{array}{l}\text { Lactococcus lactis } \\
\text { sub sp lactis }\end{array}$ & $4 a$ & 10 & 0 & 12 & 10 & 0 & 0 & 11 \\
\hline
\end{tabular}

The results of the interaction vis the advice of St. aureus and Listeria results show that among the 16 strains inhibit 7souches St.aureus (Cip20256 Bordeaux) (see fig3) strain and 10 inhibit St.aureus ATCC.602 (Tlemcen), (see fig2) strain $8 \mathrm{~b}$ has a diameter of $15 \mathrm{~mm}$, this strain was tested vis-à-vis listeria she gave a $14 \mathrm{~mm}$ diameter wells by the method of the indirect method described by Barefoot et al. (1983), that use the supernatant of the culture medium of a bacterium assumed to be inhibitory. The action of the supernatant obtained after centrifugation of the culture medium is tested against a bacterium called sensitive indicator (see fig4, Table 4).

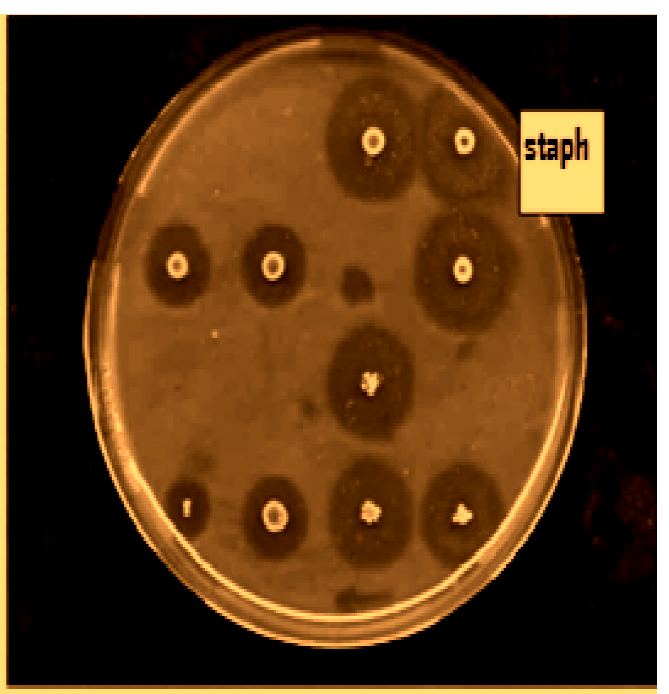

Fig.2. Interaction between de isolates and St.aureus ATCC.602(tlemcen)

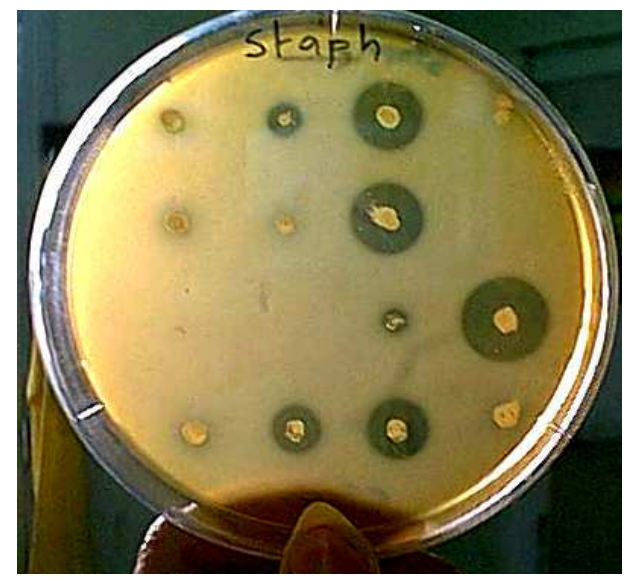

Fig.3. Interaction between de isolates and St.aureus (Cip20256 Bordeaux)

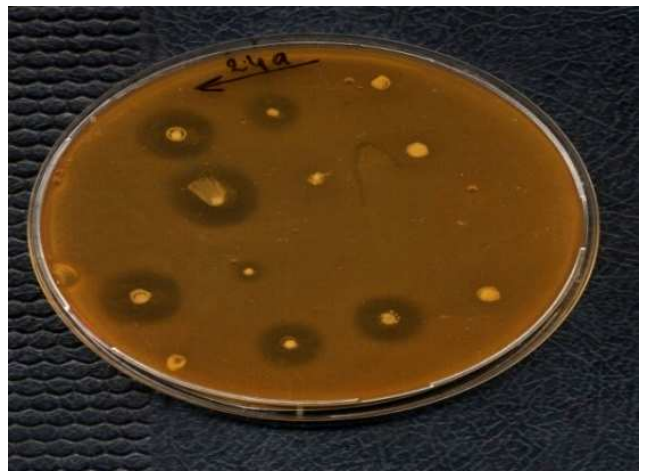

Fig.4. Interaction between isolates and indicator strain $24 a$

Strains have shown a large diameter screw the advice of St. aureus, 1b, 20b, 8b, 4a were tested agains listerie innocua. 
The results show that the supernatant adjusted $\mathrm{pH} 7$ pour strains $8 \mathrm{~b}, 20 \mathrm{~b}, 1 \mathrm{~b}$ and $4 \mathrm{a}$ gave inhibitions vis-à-vis Listeria innocua after $24 \mathrm{~h}$ of incubation at $30^{\circ}$ (see fig5)

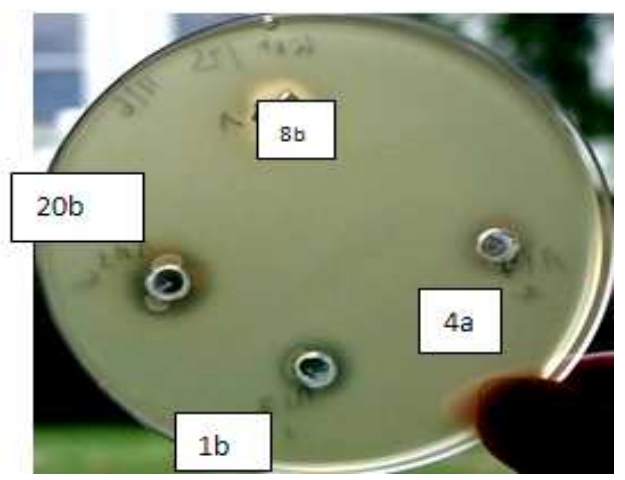

Fig.5. interaction between isolates and de listeria innocua

Determining the nature of the inhibitor: The results showed that among 48 strains tested after confrontation 768 7 strains have been selected $1 \mathrm{~b}$ strains, $8 \mathrm{~b}$ and $30 \mathrm{a}$ are presented inhibitions vis-à-vis the test strain with a diameter of 14 and $15 \mathrm{~mm}$. (Untreated supernatant) they are noted sensitivity vis-a-vis the trypsin and alpha chymotrypsin by cons. strains $2 b, 20 b, D S 15$ and $4 a$ is shown resistance vis-à-vis the proteolytic enzymes.

Table 5. Highlight interaction between lactic acid bacteria in the presence and absence of proteolytic enzyme

\begin{tabular}{|c|c|c|c|c|c|c|c|c|}
\hline Nom espèce & $\begin{array}{l}\text { Cod } \\
\text { e }\end{array}$ & $\begin{array}{l}\text { Puits } \\
\text { vide }\end{array}$ & $\begin{array}{l}\text { Try } \\
\text { psine }\end{array}$ & $\begin{array}{l}\text { Achy } \\
\text { Motrip } \\
\text { sine }\end{array}$ & $\begin{array}{l}\text { Lysoz } \\
\text { yme }\end{array}$ & $100^{\circ}$ & pH7 & $\begin{array}{l}\text { Chloro- } \\
\text { forme }\end{array}$ \\
\hline $\begin{array}{l}\text { Leuconostoc } \\
\text { mesenteroides } \\
\text { subsp.mesentero } \\
\text { ides }\end{array}$ & $2 b$ & 1,2 & 0,2 & 0,2 & 0 & 1,3 & 1,3 & 1,1 \\
\hline $\begin{array}{l}\text { Lactococcus } \\
\text { lactis sub } \\
\text { sp lactis } \\
\text { biovar } \\
\text { diacetylactis }\end{array}$ & $1 b$ & 1,5 & 0 & 0 & 0 & 1,1 & 1,3 & 1,2 \\
\hline $\begin{array}{l}\text { Lactococcus } \\
\text { lactis sub } \\
\text { sp lactis } \\
\text { biovar } \\
\text { diacetylactis }\end{array}$ & $20 \mathrm{~b}$ & 1,3 & 0,1 & 0,1 & 0 & 1,3 & 1,4 & 1,1 \\
\hline $\begin{array}{l}\text { Lactococcus } \\
\text { lactis sub } \\
\text { sp lactis } \\
\text { biovar } \\
\text { diacetylactis }\end{array}$ & $\begin{array}{l}\text { Ds } 1 \\
5\end{array}$ & 1,2 & 0,1 & 0,1 & 0 & 1,2 & 1,4 & 1,2 \\
\hline $\begin{array}{l}\text { Actococcus } \\
\text { lactis sub } \\
\text { sp lacts }\end{array}$ & $8 \mathrm{~b}$ & 1,5 & 0 & 0 & 0,2 & 1,2 & 1,5 & 1,2 \\
\hline $\begin{array}{l}\text { Lactococcus } \\
\text { lactis sub } \\
\text { sp lactis } \\
\text { biovar } \\
\text { diacetylactis }\end{array}$ & $30 a$ & 1,4 & 0 & 0 & 0 & 1,2 & 1,4 & 1,3 \\
\hline $\begin{array}{l}\text { Lactococcus } \\
\text { lactis sub } \\
\text { sp lactis }\end{array}$ & $4 a$ & 1,2 & 0,2 & 0,2 & 0 & 1,2 & 1,4 & 1,3 \\
\hline
\end{tabular}

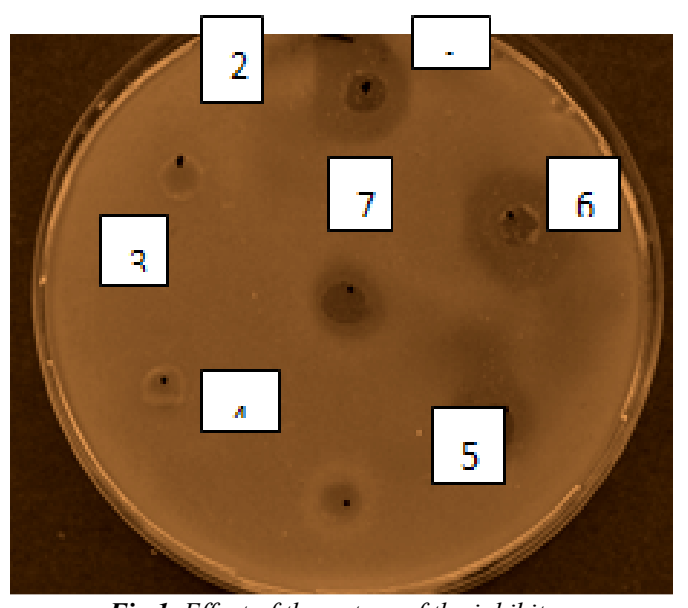

Fig.1. Effect of the nature of the inhibitor

1-Filtrat non traité 2-Filtrat + trypsine 3-filtrat + achymotrypsine 4-Filtrat+lysozyme 5-filtrat chauffée à $100^{\circ}$ 6-Filtrat ajuster à $\mathrm{pH}=7$ 7-Filtrat + chloroforme

All strains are presented for the inhibition test supernatant heated to $100^{\circ}$, and the supernatant adjusted to $\mathrm{pH}=7$. and the supernatant added chloroform.(See table5, fig 1).

For the study of the kinetics of acidificationet grows we chose the most efficient strain $8 \mathrm{~b}$ aprésenté diameter $15 \mathrm{~mm}$ agains St.aureus and $14 \mathrm{~mm}$ screws to screw listeria.

After $24 \mathrm{~h}$ of incubation, the $\mathrm{pH}$ of the producing strain $8 \mathrm{~b}$ could reach 4.53 and 5.2 for Staphylococcus aureus. An antagonism was detected in mixed culture and the $\mathrm{pH}$ was always equal to 24 hours so it has 6 results confirmed that there was a conflict between the producer strain and strain test (see Table 6 fig6) Same results were observed by (Hamama et al, 2002), $\mathrm{pH}=4.50$ after $24 \mathrm{~h}$ of incubation, 4.23 after $72 \mathrm{~h}$ of incubation $\mathrm{pH}=4.14$ after $96 \mathrm{~h}$ incubation the amount of lactic acid produced by pure cultures did not show a big difference $4.7 \mathrm{~g}$ for the producing strain ( $8 \mathrm{~b}$ ) and $3 \mathrm{~g}$ for Staphylococcus aureus after $24 \mathrm{~h}$ of incubation.

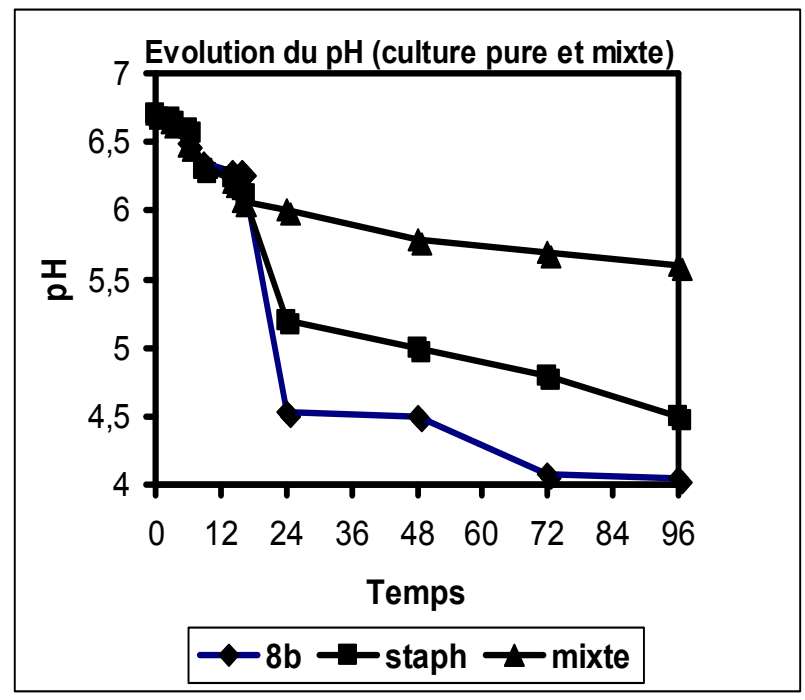

Fig.6. Evolution of $\mathrm{pH}$ as a function of time 
Table 6. Evolution of $\mathrm{pH}$ as a function of time

\begin{tabular}{|c|c|c|c|}
\hline Temps (h) & $8 b$ & St.aureus & Culture Mixte \\
\hline 0 & 6.71 & 6.70 & 6.71 \\
\hline 3 & 6.66 & 6.67 & 6.65 \\
\hline 6 & 6.48 & 6.59 & 6.47 \\
\hline 9 & 6.35 & 6.30 & 6.34 \\
\hline 14 & 6.29 & 6.24 & 6.20 \\
\hline 16 & 6.28 & 6.15 & 6.06 \\
\hline 24 & 4.53 & 5.2 & 6.00 \\
\hline 48 & 4.50 & 5.0 & 5.78 \\
\hline 72 & 4.07 & 4.8 & 5.70 \\
\hline 96 & 4.05 & 4.5 & 5.60 \\
\hline
\end{tabular}

For the mixed culture showed a decrease in the amount of lactic acid $50 \%$ which means that there is an antagonism between the two strains. (See array7, fig7).

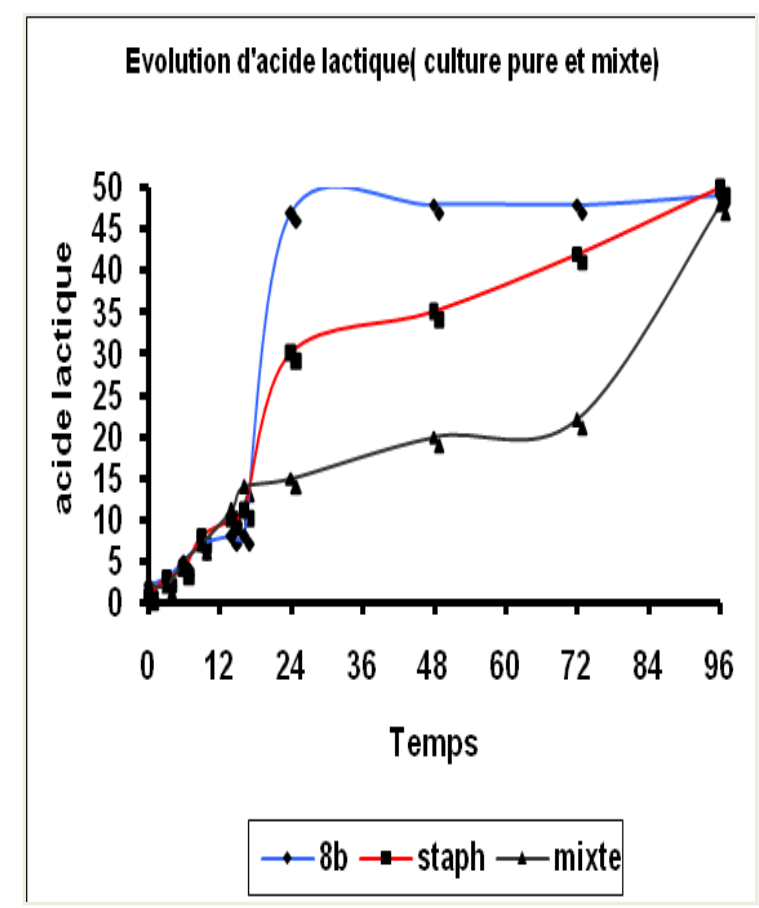

Fig.7. Evolution of lactic acid as a function of time
Table 7. Evolution of lactic acid as a function of time

\begin{tabular}{llll}
\hline & $\mathbf{8 b}$ & St.aureus & Mixed culture \\
\hline Temps(h) & Lactic acid ( $\mathbf{g}$ /) & \\
\hline 0 & 2 & 1 & 1 \\
3 & 3 & 3 & 3 \\
6 & 5 & 4 & 5 \\
9 & 7 & 8 & 7 \\
14 & 8 & 10 & 11 \\
16 & 8 & 11 & 14 \\
24 & 47 & 30 & 15 \\
48 & 48 & 33 & 20 \\
72 & 48 & 42 & 22 \\
96 & 49 & 50 & 48 \\
\hline
\end{tabular}

These results confirm what has been demonstrated by Hamama et al. (2002) in mixed culture the amount of lactic acid produced after $18 \mathrm{~h}$ preculture was $1.13 \mathrm{~g}$ after 24 hours of incubation and it was after $241.5 \mathrm{~g}$ h incubation.

For the kinetics of growth in pure culture was not observed a significant difference in the number of log unit. After $24 \mathrm{~h}$ of incubation, the number of logarithmic unit for producing strain $8 \mathrm{~b}$ was almost like her either in pure culture (10.28 log cfu g-1). Or mixed (10.77 log cfu g-1 for the test strain Staphylococcus aureus the case was different, it was observed a decrease of strain in pure culture was $9.14 \log \mathrm{cfu}$ $\mathrm{g}-1$ and $7.68 \mathrm{cfu}$ g- 1 in mixed culture after $24 \mathrm{~h}$ of incubation. (see Table 8 fig8).

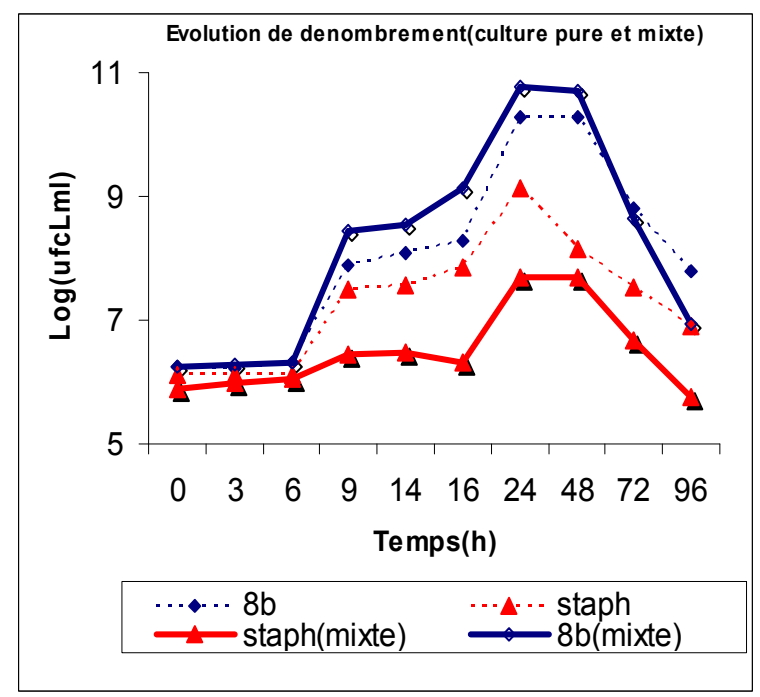

Fig.8. Evolution of the counting function of time. M123456789101 12. 
Table 8. Evolution of dénombrement as a function of time

\begin{tabular}{lllll}
\hline Temps(h) & $\begin{array}{l}\mathbf{8 b} \\
\text { (MRS) }\end{array}$ & $\begin{array}{l}\text { St.aureus } \\
\text { (chapman) }\end{array}$ & $\begin{array}{l}\text { St.aureus(Mixte) } \\
\text { (Chapman) }\end{array}$ & $\begin{array}{l}\mathbf{8 b} \text { (Mixte) } \\
\text { MRS }\end{array}$ \\
\hline 0 & 6.24 & 6.10 & 5.87 & 6.25 \\
3 & 6.20 & 6.11 & 5.99 & 6.28 \\
6 & 6.30 & 6.13 & 6.04 & 6.31 \\
9 & 7.90 & 7.48 & 6.43 & 8.44 \\
14 & 8.08 & 7.55 & 6.47 & 8.53 \\
16 & 8.27 & 7.86 & 6.30 & 9.12 \\
24 & 10.28 & 9.14 & 7.68 & 10.77 \\
48 & 10.27 & 8.14 & 7.68 & 10.71 \\
72 & 8.81 & 7.51 & 6.68 & 8.65 \\
96 & 7.79 & 6.90 & 5.74 & 6.92 \\
\hline
\end{tabular}

The growth rate of $\mu=0.73$ for $\mu=0.598 \mathrm{~b}$ strain to strain St. aureus and 0.17 for mixed culture.

The result of the random amplified polymorphic deoxyribonucleic acid (Random Amplified Polymorphic DNA) on colony using specific primers as 0PA3 allowed us to detect DNA bands corresponding to ADNr16s discomfort among strains studied, all amplicons (7a, 15a, Ma27, DS16, $1 \mathrm{~b}, 20 \mathrm{~b}$ and $30 \mathrm{a}$ have the same electrophoretic profile corresponding to a DNA fragment is a $1.5 \mathrm{Kbp}$ (see fig9). and for all primer OPH3 emplimères 18a, 27b, DS29, 25a, DS52, $4 \mathrm{a}, \mathrm{DS} 15$ has a similar profile électroforètique corresponds to a DNA fragment located at (1.5 kbp) (see Figure 10).

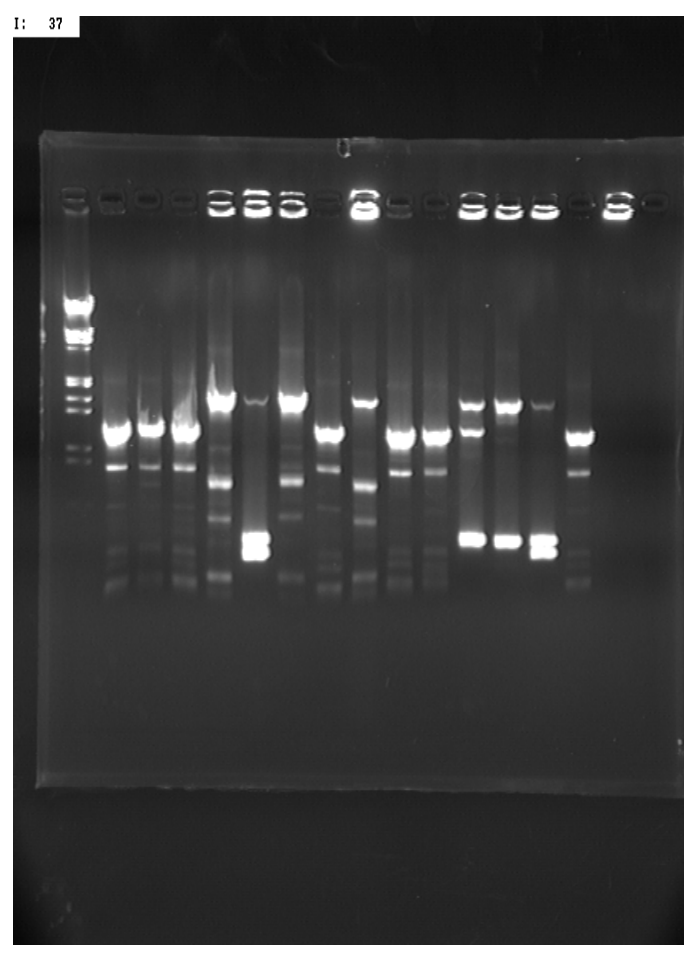

Fig.9. Following amplification assay analyzed by electrophoresis agarose gel of different strains of Lactococcus (primer OPA3). M 12345678910 111213141516

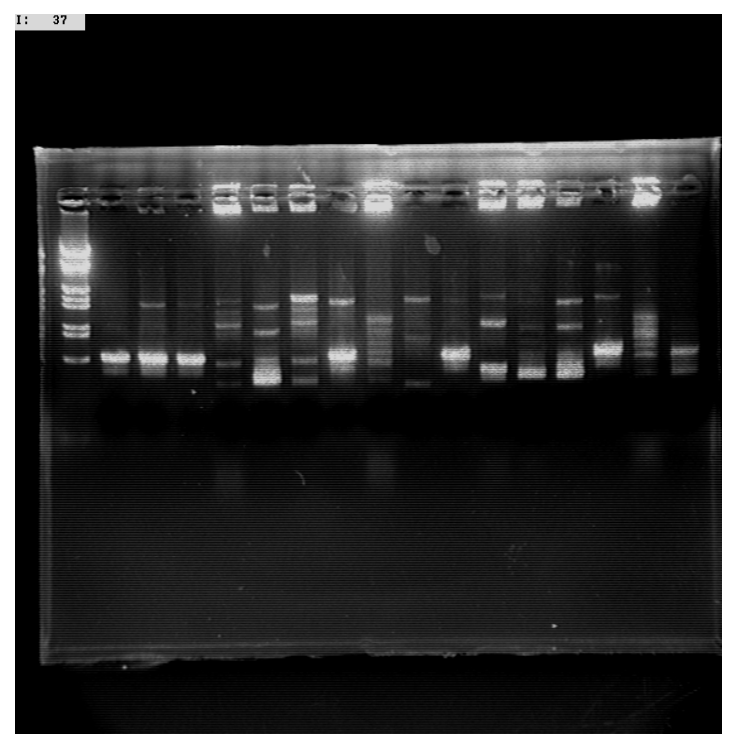

Fig.10. Following amplification assay analyzed by electrophoresis agarose gel of different strains of Lactococcus(primer OPH3). M 161718192021 222324

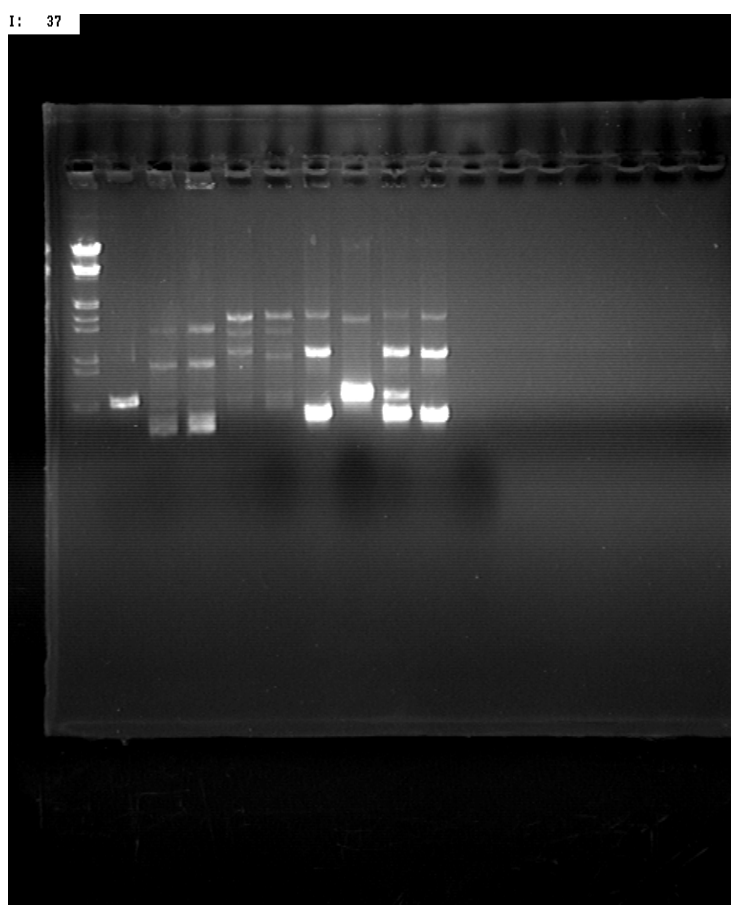

Fig.11. Following amplification assay analyzed by electrophoresis agarose gel respectful strains of the genus Enterococcus and Streptococcus and Pediococcus termophylus (primer OPA3)

These results are comparable to those found by Hamama et al, (2002) who found that the number of logarithmic unit of Staphylococcus aureus was 5.36 after $24 \mathrm{~h}$ of incubation in mixed culture, whereas this number was 7.68 after $24 \mathrm{~h}$ incubation.

According to the literature the size of the gene coding for $16 \mathrm{~S}$ rRNA in a measure lactococus $1540 \mathrm{pb}$ (Roudidre L et al, 2007) confirming that the bands represented in the electrophoretic profile correspond to fragment ADNr16s therefore studied strains belong all the kind of lactococus. 


\section{Conclusions}

Phenotypic methods may not be the final result of the identification of the bacterial species random amplification of DNA polymorfisme (RAPD) sequencing revealed 12 strains of Leuconostoc mesenteroides, 8 strains Enterococcus faecium, duran, and 4 St.thermophilus (see Table 9). These results are comparable with those found by CHANOS et al 2011 that enterococci were the most dominant in the milk medium.

Table 9. Phenotypic and molecular identification of isolates

\begin{tabular}{|c|c|c|c|}
\hline$\underline{\mathbf{N}^{\circ}}$ & code & Identification phénotypique & Séquençage PCR/RAPD \\
\hline 1 & $7 \mathrm{a}$ & L. lactis sub sp.lactisbiovardiacetylactis & Leuconostoc mesenteroides \\
\hline 2 & $15 \mathrm{a}$ & L. lactis sub sp.lactisbiovardiacetylactis & Leuconostoc mesenteroides \\
\hline 3 & $\mathrm{Ma} 27$ & L. lactis sub sp.lactis & Leuconostoc mesenteroides \\
\hline 4 & $18 \mathrm{a}$ & L. lactis sub sp.lactisbiovardiacetylactis & Leuconostoc mesenteroides \\
\hline 5 & $27 b$ & L. lactis sub sp.lactisbiovardiacetylactis & Enterococcus faecium, durans, hirae \\
\hline 6 & Ds29 & L. lactis sub sp.lactis & Leuconostoc mesenteroides \\
\hline 7 & Ds 16 & L. lactis sub sp.lactis & Leuconostoc mesenteroides \\
\hline 8 & $25 \mathrm{a}$ & L. lactis sub sp.lactisbiovardiacetylactis & Leuconostoc mesenteroides \\
\hline 9 & $1 \mathrm{~b}$ & L. lactis sub sp.lactisbiovardiacetylactis & Leuconostoc mesenteroides \\
\hline 10 & $20 \mathrm{~b}$ & L. lactis sub sp.lactisbiovardiacetylactis & Leuconostoc mesenteroides \\
\hline 11 & Ds 23 & L. lactis sub sp.lactis & Leuconostoc mesenteroides \\
\hline 12 & $4 a$ & L. lactis sub sp.lactis & Leuconostoc mesenteroides \\
\hline 13 & Ds 15 & L. lactis sub sp.lactisbiovardiacetylactis & Enterococcus faecium, durans, hirae \\
\hline 14 & $30 \mathrm{a}$ & L. lactis sub sp.lactisbiovardiacetylactis & Leuconostoc mesenteroides \\
\hline 15 & Ds53 & L. lactis sub sp.lactis & Enterococcus faecium, durans, hirae \\
\hline 16 & Ds52 & Pediococcus acidilacticci & Streptococcus thermophylus \\
\hline 17 & $8 \mathrm{~b}$ & Enterococcus sp. & Enterococcus faecium, durans, hirae \\
\hline 18 & $17 \mathrm{a}$ & Enterococcus sp. & Enterococcus faecium, durans, hirae \\
\hline 19 & $21 \mathrm{a}$ & Enterococcus sp. & Streptococcus thermophylus \\
\hline 20 & $6 a$ & Enterococcus sp. & Streptococcus thermophylus \\
\hline 21 & $7 \mathrm{~b}$ & Enterococcus sp. & Enterococcus faecium, durans, hirae \\
\hline 22 & $31 b$ & Streptococcus thermophylus & Streptococcus thermophylus \\
\hline 23 & $11 \mathrm{a}$ & Streptococcus thermophylus & Enterococcus faecium, durans, hirae \\
\hline 24 & $18 \mathrm{~b}$ & Streptococcus thermophylus & Enterococcus faecium, durans, hirae \\
\hline
\end{tabular}

Leuconostoc mesenteroides and Enterococcus faecium have summers dominant anti-Listeria species in milk samples from the western Algerian goat. Isolates had the potential of multiple bacteriocin production and do not have some important virulence.

Importance and impact of the study: The Enterococci in milk in this region of western Algeria could be partly responsible for the safety of cheese and could be useful for the production of anti-Listeria cultures protection.

The selection of strains that are potent inhibitors was based on two points: the diameter of the zone of inhibition on solid medium and the percentage of inhibition in mixed culture.

The statistical results based on student test confirmed that our results were significant confirming that the buffered medium is favorable medium for the detection of inhibitory strains. Interactions with Staphylococcus aureus showed that seven strains possess an inhibitory effect.

Strain $8 \mathrm{~b}$ gave considerable inhibition zones agains Listeria innocua and Staphylococcus aureus, the inhibition is due to an inhibitory substance knowing that the whole experience has been packaged in a buffered medium. Additional tests peroxide namely, phage, and the effect of proteolytic enzymes confirmed that the protein nature of this inhibitory substance which is a bacteriocin as defined characters Tagg et al (1976).

Phenotypic methods can not be the final result of the identification of the bacterial species random amplification of DNA polymorfisme (RAPD) sequencing revealed 12 strains of Leuconostoc mesenteroides, 8 strains Enterococcus faecium, duran, and 4 strain of St.thermophilus. These results are comparable with those found by CHANOS et al 2011 that enterococci were the most dominant in the milk medium. Importance and impact of the study: The Enterococci in milk in this region of western Algeria could be partly responsible for the safety of cheese and could be useful for the production of anti-Listeria cultures protection. Leuconostoc mesenteroides and Enterococcus faecium have summers dominant anti-Listeria species in milk samples from goats. Isolates had the potential of multiple bacteriocin production and do not have some important virulence.

Lactic acid bacteria behave as excellent ambassadors of the microbial world often maligned. They can not be reduced to their economic importance, but play an important role in the maintenance and improvement of human health.

\section{Acknowledgment}

I thank Wed Kihal Mebrouk professor at the University for its oran es senia constant availability and kindness towards me. A professor Maria URDACI ENITA de bordeaux for the time she spent to make this quality work.

\section{References}

[1] Arquès et al.Inactivation of Staphylococcus aureus in raw 
cheese by combination of high-pressure treatments and bacteriocin-producing lactic acid bacteria.J of Applied (2005) :98,254 M-260.

[2] Barefoot et al. Detection and activity of lactocin B, bacteriocin produced by Lactobacillus acidophilus. Appl.Environ.Microbio (1983) .45:1808-1815.

[3] Bayoub et al. Contribution to the study of bacteriocins produced by strains isolated from traditional fermented milk "RAIB." Biochemistry and substances Nturelles Environnement.Congrée International Biochemistry (2006) .417-420, Agadir.

[4] Complete Genome Sequence Bolotin Etal.The Of The Lactic Acid Bacterium Lactococcus Lactis Ssp. Lactis I11403. Genome Res (2001), 11 (5) :731-53.

[5] Carine Dortu and Philippe Thonart. Bacteriocins of lactic acid bacteria - characteristics and interests for food bioconservation (2008) -1 (13), 1965.

[6] CHANOS, DR-WILLIAMS.ANTI LISTERIA BACTERIOCIN-producing BACTERIA FROM RAW EWE'S MILK IN NORTHERN GREECE P. Journal of Applied Microbiology (2011) DOI: 10.1111/J.1365-2672.2011.04932.X.

[7] Davies. F and Gasson. M. Reviews of the progress of dairy science: genetics of lactic acid Bactria. J. Dairy Res, (1981) $51: 325-337$.

[8] C. De Man J, Rogosa M scarf and ME .. A medium for cultivation of lactobacilli.J.Appl. Bacteriol. (1960), $23: 130-135$.

[9] Dortu Thonart C and P. "Lactic acid bacteria: The bacteriocins of lactic acid bacteria: characteristics and interests for bioconservation food." Biotechnol. Agron. Soc. About (2009). 13 (1), 143-154.

[10] Dortu C. Isolation of a lactic acid bacterium producing the $\mathrm{G}$ and sakacin use in food matrices. Gembloux University Faculty of Agronomic Sciences, 2008, 135 p.

[11] Focus Emile C.18 PCR and sequencing of the 16S rRNA gene. Application Bacteriology (2010). Biomnis, Paris.
[12] Fleming et al, Microbiol inhibition we isolate Pediococcus from cucumber bune, Appl, Environ.Microbiol (1975), $30: 1040-1042$.

[13] Gravesena et al. Frequency of bacteriocin resistance development and associated fitness Costs in Listeria Monocytogenes dairy and food science, veterinary and agricultural university royal, rolighedsvej 30, DK-1958 frederiksberg, denmark.2011.

[14] Hamama et al. Enterotoxigenic Staphylococcus aureus Take of in the presence of nisin-producing Lactococcus lactis strain falling on manufacture of gben, a Moroccan traditional fresh cheese International Dairy Journal (2002) 12:933-938.

[15] Klaenhammer et al. Improved lysis of group N streptococci for isolation and rapid characterization of plasmid deoxyribonucleic acid. Appl.About.Microbiology(1978) 35:592-600.

[16] Kouakou Privat, Philippe Thonart.Action protective cultures: the case of lactic acid bacteria flora food junk. Biotechnol. Agron. Soc. About. 201115 (2), 339-348.

[17] Labioui.et al. selected strains of lactic acid bacteria antibacterial, Bull. Soc. Pharm. Bordeaux (2005), 144, 237-250 .

[18] Mohamadzadeh M et al. "Dendritic cell targeting of Bacillus anthracis protective antigen by Lactobacillus acidophilus Expressed mice from lethal challenge Protects" - Life Sciences (2009). Number 106 (11), pp. 4331-4336.

[19] Ramos et al. Fragmentation of lactose-protease plasmid derivatives from lactose negative Sc.lactis.et Sc.lactis subsp.diacetylactis.Ann.Microbiol (1983), 48 :387-399.

[20] S. Ananou. Evaluation of an enterocin AS-48 enriched bioactive obtenues powder by spray drying food microbiology (2010), Vol27 :58-63.

[21] Sameli al.Stability and safety and salami-a microbiological oftraditionnalGreek stydy.InternationalJournal of Food Microbiology Ecology (1998) 44pp.69-82.

[22] Touvat A. Yogurt future vaccine will be flavored Life Sciences. BBE United States (2009) N 161-electroniques.com/actualites/58592.htm. 